TRANSACTIONS OF THE

AMERICAN MATHEMATICAL SOCIETY

Volume 352, Number 9 , Pages 3971-3980

S 0002-9947(00)02602-7

Article electronically published on May 22, 2000

\title{
UNIFORM DENSITIES OF REGULAR SEQUENCES IN THE UNIT DISK
}

\author{
PETER L. DUREN, ALEXANDER P. SCHUSTER, AND KRISTIAN SEIP
}

\begin{abstract}
The upper and lower uniform densities of some regular sequences are computed. These densities are used to determine sequences of sampling and interpolation for Bergman spaces of the unit disk.
\end{abstract}

\section{INTRODUCTION}

For $0<p<\infty$, the Bergman space $A^{p}$ consists of functions $f$ analytic in the unit disk $\mathbb{D}$ with

$$
\|f\|_{p}=\left\{\frac{1}{\pi} \int_{\mathbb{D}}|f(z)|^{p} d m(z)\right\}^{1 / p}<\infty
$$

where $d m$ denotes Lebesgue area measure. Let $\Gamma=\left\{z_{k}\right\}$ be a sequence of distinct points in $\mathbb{D}$ having no accumulation points except on the boundary. We say that $\Gamma$ is a sampling sequence for $A^{p}$ if there exist positive constants $K_{1}$ and $K_{2}$ such that

$$
K_{1}\|f\|_{p}^{p} \leq \sum_{k=1}^{\infty}\left(1-\left|z_{k}\right|^{2}\right)^{2}\left|f\left(z_{k}\right)\right|^{p} \leq K_{2}\|f\|_{p}^{p}
$$

for all $f \in A^{p}$. We denote by $K_{1}(\Gamma)$ the largest constant $K_{1}$ for which the lower inequality in (1) holds. On the other hand, $\Gamma$ is an interpolation sequence for $A^{p}$ if for each sequence $\left\{w_{k}\right\}$ with

$$
\sum_{k=1}^{\infty}\left|w_{k}\right|^{p}\left(1-\left|z_{k}\right|^{2}\right)^{2}<\infty
$$

there is an $f \in A^{p}$ such that $f\left(z_{k}\right)=w_{k}$ for all $k$.

By a standard argument involving the closed graph theorem, if $\Gamma$ is a set of interpolation, there is a constant $M(\Gamma)$ such that the interpolation problem can be solved by a function satisfying

$$
\|f\|_{p}^{p} \leq M(\Gamma) \sum_{k}\left|w_{k}\right|^{p}\left(1-\left|z_{k}\right|^{2}\right)^{2} .
$$

It is easily verified that both sampling and interpolation sequences are conformally invariant, preserved under Möbius transformations of the disk onto itself.

The sampling and interpolation sequences for $A^{2}$ were described completely in [8], and in [4] and [5] the methods were used to extend the results to $1 \leq p<\infty$. The descriptions are given in terms of certain densities. In order to state the theorems, it is necessary first to introduce some terminology.

Received by the editors July 10, 1998.

2000 Mathematics Subject Classification. Primary 30H05, 46E15. 
For $z, \zeta \in \mathbb{D}$, the pseudohyperbolic metric is

$$
\rho(z, \zeta)=\left|\phi_{\zeta}(z)\right|, \quad \text { where } \phi_{\zeta}(z)=\frac{\zeta-z}{1-\bar{\zeta} z} .
$$

The pseudohyperbolic disk with centre $\alpha$ and radius $r$ is

$$
\Delta(\alpha, r)=\{z \in \mathbb{D}: \rho(z, \alpha)<r\} .
$$

The hyperbolic area of a measurable set $\Omega \subset \mathbb{D}$ is

$$
A(\Omega)=\frac{1}{\pi} \int_{\Omega} \frac{d m(z)}{\left(1-|z|^{2}\right)^{2}} .
$$

It is easily seen to be conformally invariant. Thus the hyperbolic area of a pseudohyperbolic disk is

$$
A(\Delta(\alpha, r))=A(\Delta(0, r))=\frac{r^{2}}{1-r^{2}} .
$$

A sequence $\Gamma$ is uniformly discrete if

$$
\delta(\Gamma)=\inf _{j \neq k} \rho\left(z_{j}, z_{k}\right)>0
$$

For a uniformly discrete sequence $\Gamma$, let $n_{\Gamma}(\alpha, r)$ denote the number of points of $\Gamma$ which lie in $\Delta(\alpha, r)$. Consider the expression

$$
D_{\Gamma}(\alpha, r)=\frac{\int_{0}^{r} n_{\Gamma}(\alpha, s) d s}{\log \frac{1+r}{1-r}} .
$$

Since

$$
\log \frac{1+r}{1-r}=2 \int_{0}^{r}\{A(\Delta(\alpha, s))+1\} d s,
$$

the quantity $D_{\Gamma}(\alpha, r)$ may be viewed as the average number number of points in pseudohyperbolic disks centred at $\alpha$, per unit of hyperbolic area. The upper and lower uniform densities of $\Gamma$ are defined, respectively, by

$$
D^{+}(\Gamma)=\limsup _{r \rightarrow 1} \sup _{\alpha \in \mathbb{D}} D_{\Gamma}(\alpha, r)
$$

and

$$
D^{-}(\Gamma)=\liminf _{r \rightarrow 1} \inf _{\alpha \in \mathbb{D}} D_{\Gamma}(\alpha, r) .
$$

We can now state the theorems that describe sampling and interpolation sequences in Bergman spaces.

Theorem A. A sequence $\Gamma$ of distinct points in the disk is a sampling sequence for $A^{p}$ if and only if it is a finite union of uniformly discrete sets and it contains a uniformly discrete subsequence $\Gamma^{\prime}$ for which $D^{-}\left(\Gamma^{\prime}\right)>\frac{1}{p}$.

Theorem B. A sequence $\Gamma$ of distinct points in the disk is an interpolation sequence for $A^{p}$ if and only if $\Gamma$ is uniformly discrete and $D^{+}(\Gamma)<\frac{1}{p}$.

Interpolation sequences for Hardy spaces are described by a condition known as uniform separation. For Bergman spaces the interpolation sequences have a similar description [6] in which a canonical divisor plays the role of the Blaschke product.

Theorems A and B will be effective in describing specific sampling and interpolation sequences only insofar as the densities can be computed. The calculation of densities can be quite difficult, but answers are known in some instances. In [7] a 
regular sequence with two parameters is introduced, and by direct arguments it is classified as a sampling or interpolation sequence for $A^{2}$, depending on the choice of parameters. In [3], the techniques of [7] are used to make a similar analysis of a sequence constructed in [9]. The densities of these sequences can then be inferred by comparing the results with Theorems $\mathrm{A}$ and $\mathrm{B}$.

The purpose of this article is to calculate the densities of these same sequences directly. We will perform the calculation only for the sequence of [9], since the method will apply to the sequence of [7] with minor modifications. Our main point is to show that the numerical densities can be extracted directly from the construction of the sequence, by estimates similar to those which in 9 led to the existence of a function vanishing on the sequence and with a particularly regular asymptotic behavior. As a corollary, we also find the densities of sequences investigated by Horowitz [1] and Luecking [2].

\section{A REGULAR SEQUENCE IN THE DISK}

For the calculation of densities it is more efficient to use the following equivalent definition. (See [5] for a proof that the definitions are equivalent.) Again let $\Gamma$ be a uniformly discrete sequence in $\mathbb{D}$. For a fixed constant $c_{0}, 0<c_{0}<1$, let

$$
E_{\Gamma}(\alpha, r)=\frac{\sum_{c_{0}<\left|\phi_{\alpha}\left(z_{k}\right)\right|<r} \log \frac{1}{\left|\phi_{\alpha}\left(z_{k}\right)\right|}}{\log \left(\frac{1}{1-r}\right)}
$$

Then

$$
D^{+}(\Gamma)=\limsup _{r \rightarrow 1} \sup _{\alpha \in \mathbb{D}} E_{\Gamma}(\alpha, r)
$$

and

$$
D^{-}(\Gamma)=\liminf _{r \rightarrow 1} \inf _{\alpha \in \mathbb{D}} E_{\Gamma}(\alpha, r)
$$

It is easy to see that these definitions do not depend on $c_{0}$.

We turn now to a sequence previously constructed in [9]. For a fixed constant $a>0$, let

$$
d \nu(z)=\frac{a d m(z)}{\left(1-|z|^{2}\right)^{2}}
$$

denote the corresponding multiple of hyperbolic area measure. Divide the disk into a union of disjoint annuli

$$
R_{n}=\left\{z: t_{n-1} \leq|z|<t_{n}\right\}, \quad n=1,2,3, \ldots
$$

where the radii $t_{n}$ are defined inductively by $t_{0}=0$ and $\nu\left(R_{n}\right)=2^{n-1}$. A calculation shows that

$$
\frac{1}{1-t_{n}^{2}}=\frac{2^{n}+\pi a-1}{\pi a}
$$

so that

$$
t_{n}=1-\pi a 2^{-n-1}+O\left(2^{-2 n}\right) .
$$

In particular, $t_{n} \rightarrow 1$ as $n \rightarrow \infty$. 
Next divide each annulus $R_{n}$ into $2^{n-1}$ cells $Q_{n j}$, by placing radial segments at angles $j 2^{-n+2} \pi, j=1,2, \ldots, 2^{n-1}$. Then all cells have equal area $\nu\left(Q_{n j}\right)=1$. Let

$$
\zeta_{n j}=\int_{Q_{n j}} z d \nu(z)
$$

denote the hyperbolic centre of mass of the cell $Q_{n j}$. A calculation shows that

$$
\left|\zeta_{n j}\right|=1-2^{-n}(\pi a \log 2)+O\left(2^{-2 n}\right)
$$

so that $\zeta_{n j} \in Q_{n j}$ at least for all $n$ sufficiently large. Finally, we define our sequence $\Gamma=\left\{z_{k}\right\}$ to be an enumeration of the points $\zeta_{n j}$. We will show that $\Gamma$ is uniformly discrete and will establish the following

\section{Main Result.}

$$
D^{-}(\Gamma)=D^{+}(\Gamma)=\frac{a \pi}{2}
$$

For the proof, we define the discrete integer-valued "counting measure"

$$
\eta=\sum_{k} \delta_{z_{k}}
$$

where $\delta_{z}$ is the Dirac measure located at $z$. For $\alpha \in \mathbb{D}$ and $c_{0}<r<1$, consider the pseudohyperbolic annulus

$$
\Omega(\alpha, r)=\left\{z \in \mathbb{D}: c_{0}<\rho(\alpha, z)<r\right\} .
$$

We intend to prove

\section{Theorem 1.}

$$
\sup _{\alpha \in \mathbb{D}, r<1}\left|\int_{\Omega(\alpha, r)} \log \right| \phi_{\alpha}(z)|d(\nu(z)-\eta(z))|<\infty
$$

Deferring the proof of Theorem 1, let us observe first that it implies the main result. By a change of variables argument and the identity

we obtain

$$
\left|\phi_{\alpha}^{\prime}(z)\right|^{2}=\frac{\left(1-\left|\phi_{\alpha}(z)\right|^{2}\right)^{2}}{\left(1-|z|^{2}\right)^{2}}
$$

$$
\begin{aligned}
\int_{\Omega(\alpha, r)} \log \left|\phi_{\alpha}(z)\right| d \nu(z) & =a \int_{c_{0}<|w|<r} \log |w| \frac{d m(w)}{\left(1-|w|^{2}\right)^{2}}=2 a \pi \int_{c_{0}}^{r} \frac{s \log s d s}{\left(1-s^{2}\right)^{2}} \\
& =-\frac{a \pi}{2} \log \frac{1}{1-r}+o\left(\log \frac{1}{1-r}\right) .
\end{aligned}
$$

On the other hand,

$$
\int_{\Omega(\alpha, r)} \log \left|\phi_{\alpha}(z)\right| d \eta(z)=\sum_{c_{0}<\left|\phi_{\alpha}\left(z_{k}\right)\right|<r} \log \left|\phi_{\alpha}\left(z_{k}\right)\right|=-\log \frac{1}{1-r} E_{\Gamma}(\alpha, r) .
$$

These two formulas are now combined to give

$$
\int_{\Omega(\alpha, r)} \log \left|\phi_{\alpha}(z)\right| d(\nu(z)-\eta(z))=\left(E_{\Gamma}(\alpha, r)-\frac{a \pi}{2}\right) \log \frac{1}{1-r}+o\left(\log \frac{1}{1-r}\right) .
$$

Thus the boundedness of the integral implies that $D^{-}(\Gamma)=D^{+}(\Gamma)=\frac{a \pi}{2}$.

We now turn to the proof of Theorem 1. A major part of it coincides with the proof of Theorem 2 of [9], but we elaborate the details for the convenience of the reader. 
We begin by making some preliminary observations. We will denote the pseudohyperbolic diameter of a set $E \subseteq \mathbb{D}$ by

$$
d(E)=\sup _{z, \zeta \in E} \rho(z, \zeta) .
$$

Lemma 1. (a) The sequence $\Gamma$ is uniformly discrete.

(b) The pseudohyperbolic diameters of the cells $Q_{n j}$ are uniformly bounded: $d\left(Q_{n j}\right) \leq K$ for some constant $K<1$.

Proof. Calculations based on the asymptotic formulas for $\left|\zeta_{n j}\right|$ and $t_{n}$ show that there exist radii $r_{1}$ and $r_{2}$ such that

$$
\Delta\left(\zeta_{n j}, r_{1}\right) \subseteq Q_{n j} \subseteq \Delta\left(\zeta_{n j}, r_{2}\right)
$$

at least for all $n$ sufficiently large. From this the lemma follows immediately.

Lemma 2. There is a constant $C$ such that if a and belong to the same cell $Q_{n j}$, then

$$
\left|\frac{1-\bar{a} z}{1-\bar{b} z}\right| \leq C
$$

for all $z \in \mathbb{D}$.

Proof. Note first that

$$
\left|\frac{1-\bar{a} z}{1-\bar{b} z}\right|^{2}=\frac{1-|a|^{2}}{1-|b|^{2}} \frac{1-\rho^{2}(b, z)}{1-\rho^{2}(a, z)} .
$$

The first factor can be bounded using the estimates for $1-t_{n}^{2}$ given by (2). The second factor will be handled as follows: Note that

$$
\rho(a, z) \leq \frac{\rho(b, z)+\rho(a, b)}{1+\rho(b, z) \rho(a, b)} .
$$

Therefore,

$$
\begin{aligned}
\frac{1-\rho(b, z)}{1-\rho(a, z)} & \leq \frac{1-\rho(b, z)}{1-\frac{\rho(b, z)+\rho(a, b)}{1+\rho(b, z) \rho(a, b)}}=\frac{(1-\rho(b, z))(1+\rho(b, z)) \rho(a, b)}{(1-\rho(b, z))(1-\rho(a, b))} \\
& \leq \frac{2}{1-\rho(a, b)} \leq \frac{2}{1-K}
\end{aligned}
$$

The last inequality follows from the fact that $d\left(Q_{n j}\right) \leq K$ for all $k$. This completes the proof of the lemma.

We now proceed with the proof of Theorem 1 . Denote by $\Omega_{1}(\alpha, r)$ the union of the cells $Q_{n j}$ which are entirely contained in $\Omega(\alpha, r)$ and write

$$
\Omega_{2}(\alpha, r)=\Omega(\alpha, r) \backslash \Omega_{1}(\alpha, r) .
$$

Our first task is to estimate the part of the integral in (3) taken over $\Omega_{1}(\alpha, r)$. To this end, define

$$
L(z)=\log \left(\frac{z-\alpha}{1-\bar{\alpha} z}\right)
$$

Integration by parts yields

$$
L(z)=L\left(z_{k}\right)+L^{\prime}\left(z_{k}\right)\left(z-z_{k}\right)+\int_{z_{k}}^{z} L^{\prime \prime}(w)(z-w) d w .
$$


Denoting by $Q_{k}$ the cell containing $z_{k}$, we apply this equation to obtain

$$
\int_{Q_{k}} \log \left|\frac{z-\alpha}{1-\bar{z} \alpha}\right| d(\nu(z)-\eta(z))=\operatorname{Re} \int_{Q_{k}}\left(\int_{z_{k}}^{z} L^{\prime \prime}(w)(z-w) d w\right) d \nu(z) .
$$

Here we have used the facts that

$$
\begin{gathered}
\int_{Q_{k}} d(\nu(z)-\eta(z))=1-1=0 \\
\int_{Q_{k}} z d(\nu(z)-\eta(z))=z_{k}-z_{k}=0
\end{gathered}
$$

and

$$
\operatorname{Re} \int_{Q_{k}}\left(\int_{z_{k}}^{z} L^{\prime \prime}(w)(z-w) d w\right) d \eta(z)=\operatorname{Re} \int_{z_{k}}^{z_{k}} L^{\prime \prime}(w)(z-w) d w=0 .
$$

To estimate the right-hand side of (4) we make the explicit calculation

$$
L^{\prime \prime}(w)=\frac{\left(1-|\alpha|^{2}\right)(\bar{\alpha}(w-\alpha)-(1-\bar{\alpha} w))}{(1-\bar{\alpha} w)^{2}(w-\alpha)^{2}} .
$$

But $Q_{k} \subseteq \Omega(\alpha, r)$ and so $c_{0}<\rho(\alpha, w)<r$ for all $w \in Q_{k}$. This implies that

$$
\begin{aligned}
\left|L^{\prime \prime}(w)\right| & \leq\left(1-|\alpha|^{2}\right)\left(\frac{1}{|w-\alpha||1-\bar{\alpha} w|^{2}}+\frac{1}{|w-\alpha|^{2}|1-\bar{\alpha} w|}\right) \\
& \leq\left(1-|\alpha|^{2}\right)\left(\frac{1}{c_{0}|1-\bar{\alpha} w|^{3}}+\frac{1}{c_{0}^{2}|1-\bar{\alpha} w|^{3}}\right) \\
& \leq \frac{2\left(1-|\alpha|^{2}\right)}{c_{0}^{2}|1-\bar{\alpha} w|^{3}} .
\end{aligned}
$$

Therefore, an application of Lemma 2 with $a=w$ and $b=z$ yields

$$
\begin{aligned}
\left|\int_{z_{k}}^{z} L^{\prime \prime}(w)(z-w) d w\right| & \leq C\left(1-|\alpha|^{2}\right) \int_{z_{k}}^{z} \frac{|z-w|}{|1-\bar{\alpha} w|^{3}}|d w| \\
& \leq C \frac{\left(1-|\alpha|^{2}\right)}{|1-\bar{\alpha} z|^{3}} \int_{z_{k}}^{z}|z-w||d w| \\
& \leq C \frac{\left(1-|\alpha|^{2}\right)}{|1-\bar{\alpha} z|^{3}} \sup _{w \in Q_{k}}|w-z|^{2} .
\end{aligned}
$$

But if $w \in Q_{k}$, we use Lemma 2 with $a=w, b=z$ and $z=z$ to observe that

$$
|w-z| \leq|1-\bar{w} z| \leq C\left(1-|z|^{2}\right)
$$

Therefore,

$$
\left|\int_{z_{k}}^{z} L^{\prime \prime}(w)(z-w) d w\right| \leq C \frac{\left(1-|\alpha|^{2}\right)\left(1-|z|^{2}\right)^{2}}{|1-\bar{\alpha} z|^{3}}
$$

It follows that

$$
\left|\sum_{Q_{k} \subseteq \Omega_{1}(\alpha, r)} \int_{Q_{k}} \log \right| \phi_{\alpha}(z) \mid\left(d \nu(z)-d(\eta(z)) \mid \leq C\left(1-|\alpha|^{2}\right) \int_{\mathbb{D}} \frac{1}{|1-\bar{\alpha} z|^{3}} d m(z) .\right.
$$

A computation shows that the right-hand side is bounded independently of $\alpha$. In other words, the part of the integral in (3) over $\Omega_{1}(\alpha, r)$ is bounded.

We now show that the part of the integral taken over $\Omega_{2}(\alpha, r)$ is bounded. We remarked earlier that the definitions of our densities $D^{-}(\Gamma)$ and $D^{+}(\Gamma)$ do not 
depend on $c_{0}$. We now fix such a $c_{0}$. First, we choose $L$ such that $K<L<1$, where $K$ is the constant from Lemma 1 . Next we pick $c_{0}<1$ such that

$$
\frac{2 L+c_{0}+L^{2} c_{0}}{1+2 L c_{0}+L^{2}}<1
$$

By the definition of the densities, it is enough to consider values of $r$ close to 1 . We are thus allowed to restrict ourselves to

$$
r>\frac{2 L+c_{0}+L^{2} c_{0}}{1+2 L c_{0}+L^{2}} .
$$

Let

$$
c=\frac{L+c_{0}}{1+L c_{0}} \quad \text { and } \quad h(r)=\frac{r-L}{1-r L} .
$$

(5) and (6) imply that

$$
c_{0}<c<h(r)<r .
$$

We will show that

$$
\Omega_{2}(\alpha, r) \cap\{z: c<\rho(\alpha, z)<h(r)\}=\phi .
$$

By Möbius invariance, we may assume that $\alpha=0$. To prove (7), it thus suffices to show

$$
\rho\left(c, c_{0}\right)>K
$$

and

$$
\rho(h(r), r)>K
$$

Since $L>K,(8)$ and (9) follow immediately from (6).

By (7) we see that

$$
\begin{aligned}
& \sup _{\alpha \in \mathbb{D}, r<1}\left|\int_{\Omega(\alpha, r)} \log \right| \phi_{\alpha}(z) \mid(d \nu(z)-d(\eta(z)) \mid \\
& \leq\left|\int_{c_{0}<\rho(\alpha, z)<c} \log \right| \phi_{\alpha}(z) \mid(d \nu(z)-d(\eta(z)) \mid \\
& \quad+\left|\int_{h(r)<\rho(\alpha, z)<r} \log \right| \phi_{\alpha}(z) \mid(d \nu(z)-d(\eta(z)) \mid \\
& =I_{1}+I_{2} .
\end{aligned}
$$

To prove the boundedness of $I_{1}$, we require the following well-known result, the proof of which may be found in [5].

Lemma 3. Let $\Lambda$ be a uniformly discrete sequence of points in $\mathbb{D}$. There is a constant $A$, depending only on $\delta(\Lambda)$, such that

$$
n_{\Lambda}(\alpha, r) \leq \frac{A}{1-r}
$$

for all $r<1$ and all $\alpha \in \mathbb{D}$. 
By Lemma 3 and standard estimates, we then have

$$
\begin{aligned}
I_{1} & \leq a\left|\int_{c_{0}<\rho(\alpha, z)<c} \log \right| \phi_{\alpha}(z)\left|\frac{d m(z)}{\left(1-|z|^{2}\right)^{2}}\right|+\sum_{c_{0}<\left|\phi_{\alpha}\left(z_{k}\right)\right|<c}|\log | \phi_{\alpha}\left(z_{k}\right)|| \\
& \leq \frac{\pi a c^{2}\left|\log c_{0}\right|}{1-c^{2}}+\left|\log c_{0}\right| n_{\Gamma}(\alpha, c) \\
& \leq \frac{\pi a c^{2}\left|\log c_{0}\right|}{1-c^{2}}+\frac{A\left|\log c_{0}\right|}{1-c} .
\end{aligned}
$$

On the other hand,

$$
\begin{aligned}
I_{2} & \leq a\left|\int_{h(r)<\rho(\alpha, z)<r} \log \right| \phi_{\alpha}(z)\left|\frac{d m(z)}{\left(1-|z|^{2}\right)^{2}}\right|+\sum_{h(r)<\left|\phi_{\alpha}\left(z_{k}\right)\right|<r}|\log | \phi_{\alpha}\left(z_{k}\right)|| \\
& =I_{3}+I_{4} .
\end{aligned}
$$

But

$$
I_{3}=2 \pi a \int_{h(r)}^{r} \frac{s|\log s| d s}{\left(1-s^{2}\right)^{2}} \leq 2 \pi a \int_{h(r)}^{r} \frac{s d s}{1-s^{2}} \leq \pi a \log (1+L) .
$$

To bound $I_{4}$, we require the following result used in 8 . The version below may be found in [5].

Lemma 4. Let $\Lambda$ be uniformly discrete. There is a constant $B$, depending only on $t \in \mathbb{R}, \delta(\Lambda)$ and $\epsilon<\delta(\Lambda)$, such that

$$
\sum_{\lambda_{k} \in \Lambda \cap \Omega}\left(1-\left|\lambda_{k}\right|^{2}\right)^{t}\left|f\left(\lambda_{k}\right)\right|^{2} \leq B \int_{\rho(z, \Omega)<\epsilon}|f(z)|^{2}\left(1-|z|^{2}\right)^{t-2} d m(z)
$$

for all measurable subsets $\Omega$ of $\mathbb{D}$ and all $f$ analytic in $\mathbb{D}$.

We apply this lemma to $t=1, f(z) \equiv 1$ and $\Omega=\{z: h(r) \leq|z| \leq r\}$ to obtain

$\sum_{h(r) \leq\left|\phi_{\alpha}\left(z_{k}\right)\right| \leq r}|\log | \phi_{\alpha}\left(z_{k}\right)|| \leq \sum_{h(r) \leq\left|\phi_{\alpha}\left(z_{k}\right)\right| \leq r}\left(1-\left|\phi_{\alpha}\left(z_{k}\right)\right|^{2}\right) \leq B \int_{\rho(z, \Omega)<\epsilon} \frac{d m(z)}{1-|z|^{2}}$

But $\rho(z, \Omega)<\epsilon$ if and only if

$$
\frac{h(r)-\epsilon}{1-\epsilon h(r)}<|z|<\frac{r+\epsilon}{1+r \epsilon}
$$

Therefore,

$$
\begin{aligned}
I_{4} & \leq 2 \pi \int_{\frac{h(r)-\epsilon}{1-\epsilon h(r)}}^{\frac{r+\epsilon}{1+r \epsilon}} \frac{s d s}{1-s^{2}} \\
& \leq 2 \pi \int_{\frac{h(r)-\epsilon}{1-\epsilon h(r)}}^{\frac{r+\epsilon}{1+r \epsilon}} \frac{d s}{1-s} \\
& =2 \pi \log \left(\frac{(1+\epsilon)(1+r \epsilon)}{(1-\epsilon h(r))(1-\epsilon)(1-r)}\right) \\
& \leq 2 \pi \log \left(\frac{(1+\epsilon)^{2}}{(1-\epsilon)^{2}} \frac{1+L}{1-L}\right) .
\end{aligned}
$$


This shows that the part of the integral in (3) over $\Omega_{2}(\alpha, r)$ is bounded, thus completing the proof of Theorem 1 .

As a consequence of our work, we are able to compute the density of the sequence of zeroes of the infinite product

$$
f(z)=\prod_{n=1}^{\infty}\left(1-b z^{2^{n}}\right), \quad b>1,
$$

introduced by Horowitz 1 to study $A^{p}$ zero-sets. This sequence $H$ has $2^{n}$ equally spaced points on the circle $|z|=b_{n}=(1 / b)^{2^{-n}}, \quad n=1,2, \ldots$ Luecking [2] constructs a similar sequence $\Lambda$ by placing $2^{n}$ equally spaced points on the circle $|z|=r_{n}=1-\gamma 2^{-n}, n=N, N+1, \ldots$, where $\gamma$ is a positive constant and $2^{N}>\gamma$. (Here each group is aligned to include the point $z=r_{n}$.)

Corollary 1. The Horowitz sequence $H$ has density

$$
D^{-}(H)=D^{+}(H)=\frac{\log b}{\log 2}
$$

Luecking's sequence $\Lambda$ has density

$$
D^{-}(\Lambda)=D^{+}(\Lambda)=\frac{\gamma}{\log 2}
$$

Proof. Let $\Gamma=\left\{\zeta_{n j}\right\}$ be the sequence constructed earlier in the paper and let $Z$ be obtained by placing $2^{n-1}$ points on the circle of radius $s_{n}$, where $s_{n}$ is defined by $1-s_{n}=2^{-n} \pi a \log 2$.

Recall that

$$
1-\left|\zeta_{n, j}\right|=2^{-n} \pi a \log 2+O\left(2^{-2 n}\right)
$$

We can therefore think of $\Gamma$ as a sequence obtained by placing $2^{n-1}$ points on the circle of radius $v_{n}$, which is defined by $1-v_{n}=2^{-n} \pi a \log 2+O\left(2^{-2 n}\right)$. At this point we require the following result proved in [4] and [5].

Lemma 5. Let $\Gamma$ be uniformly discrete. There are constants $\delta_{0}$ and $C_{0}$, depending only on $\delta(\Gamma)$, such that

$$
\left|K_{1}(\Gamma)^{\frac{1}{p}}-K_{1}\left(\Gamma^{\prime}\right)^{\frac{1}{p}}\right| \leq C_{0}\left[\Gamma, \Gamma^{\prime}\right]
$$

and

$$
\left|M(\Gamma)^{-\frac{1}{p}}-M\left(\Gamma^{\prime}\right)^{-\frac{1}{p}}\right| \leq C_{0}\left[\Gamma, \Gamma^{\prime}\right]
$$

for every sequence $\Gamma^{\prime}$ with $\left[\Gamma, \Gamma^{\prime}\right]<\delta_{0}$.

Here $\left[\Gamma, \Gamma^{\prime}\right]$ is the Fréchet distance between $\Gamma$ and $\Gamma^{\prime}$ with respect to $\rho$. The precise statement of the lemma holds only when $p \geq 1$, but a similar result is true for $0<p<1$. See $[5$ for details. It follows from Lemma 5 that the maps $\Gamma \mapsto D^{-}(\Gamma)$ and $\Gamma \mapsto D^{+}(\Gamma)$ are continuous if the space of sequences is endowed with the Fréchet metric.

One can check easily that $\rho\left(s_{n}, v_{n}\right) \rightarrow 0$ and so we may apply Lemma 5 to show that

$$
D^{-}(Z)=D^{+}(Z)=D^{-}(\Gamma)=D^{+}(\Gamma)=\frac{\pi a}{2}
$$


Note that $\Lambda$ can also be obtained by placing $2^{n-1}$ points on $u_{n}=r_{n-1}$; that is, $1-u_{n}=2^{-n} 2 \gamma$. Thus, $\Lambda=Z$ if $2 \gamma=\pi a \log 2$, and so

$$
D^{-}(\Lambda)=D^{+}(\Lambda)=\frac{\pi a}{2}=\frac{\gamma}{\log 2} .
$$

Since $\rho\left(r_{n}, b_{n}\right) \rightarrow 0$ if we choose $\gamma=\log b$, we again apply Lemma 5 to show that

$$
D^{-}(H)=D^{+}(H)=\frac{\log b}{\log 2} .
$$

Luecking [2] has found that $\Lambda$ is an $A^{p}$ zero-set if and only if $p \gamma<\log 2$. Horowitz [1] showed that the infinite product $f$ belongs to $A^{p}$, hence that $H$ is an $A^{p}$ zeroset, if $p \log b<\log 2$. For $p \log b>\log 2$ he proved that $H$ is not an $A^{p}$ zero-set. Luecking has now sharpened the result to show that $H$ is not an $A^{p}$ zero-set if $p \log b=\log 2$. Appealing to Theorems $\mathrm{A}$ and $\mathrm{B}$, we can now round out the picture as follows.

Corollary 2. (a) The Horowitz sequence $H$ is an interpolation sequence for $A^{p}$ if and only if $p \log b<\log 2$. It is a sampling sequence if and only if $p \log b>\log 2$. When $p \log b=\log 2$ it is neither a zero-set nor a sampling sequence.

(b) The sequence $\Lambda$ is an interpolation sequence for $A^{p}$ if and only if $p \gamma<\log 2$. It is a sampling sequence if and only if $p \gamma>\log 2$. When $p \gamma=\log 2$ it is neither a zero-set nor a sampling sequence.

\section{REFERENCES}

1. C. Horowitz, Zeros of functions in the Bergman spaces, Duke Math. J. 41 (1974), 693-710. MR 55:681

2. D. Luecking, Zero sequences for Bergman spaces, Complex Variables Theory Appl. 30 (1996), 345-362. MR 97g:30007

3. A. Schuster, Sets of sampling and interpolation in Bergman spaces, Proc. Amer. Math. Soc. 125 (1997), 1717-1725. MR 97g:46029

4. A. Schuster, Sampling and interpolation in Bergman spaces, Ph.D. thesis, University of Michigan (1997).

5. A. Schuster, On Seip's description of sampling sequences for Bergman spaces, Complex Variables Theory Appl. (to appear).

6. A. Schuster and K. Seip, A Carleson-type condition for interpolation in Bergman spaces, J. Reine Angew. Math. 497 (1998), 223-233. MR 99f:46034

7. K. Seip, Regular sets of sampling and interpolation for weighted Bergman spaces, Proc. Amer. Math. Soc. 117 (1993), 213-220. MR 93:30051

8. K. Seip, Beurling type density theorems in the unit disk, Invent. Math. 113 (1994), 21-39. MR 94g:30033

9. K. Seip, On Korenblum's density condition for the zero sequences of $A^{-\alpha}$, J. Anal. Math. 67 (1995), 307-322. MR 97c:30044 1109

Department of Mathematics, University of Michigan, Ann Arbor, Michigan 48109-

E-mail address: duren@math.lsa.umich.edu

Department of Mathematics, San Francisco State University, San Francisco, CaliFORNIA 94132-4163

E-mail address: schuster@sfsu.edu

Department of Mathematical Sciences, Norwegian University of Science and TechNOLOGY, N-7034 TRONDHEIM, NORWAY

E-mail address: seip@math.ntnu.no 